\title{
Desigualdades regionais na mortalidade por câncer de colo de útero no Brasil: tendências e projeções até o ano 2030
}

\author{
Regional inequalities in cervical cancer mortality in Brazil: \\ trends and projections through to 2030
}

Isabelle Ribeiro Barbosa ${ }^{1}$

Dyego Leandro Bezerra de Souza ${ }^{1}$

María Milagros Bernal ${ }^{2}$

Iris do Céu Clara Costa ${ }^{3}$

${ }^{1}$ Programa de PósGraduação em Saúde Coletiva, Universidade Federal do Rio Grande do Norte (UFRN). Av. Senador Salgado Filho 1787, Lagoa Nova. 59010-000 Natal RN. isabelleribeiro@oi.com.br

${ }^{2}$ Universidad de Zaragoza.

${ }^{3}$ Departamento de

Odontologia, UFRN.
Abstract The scope of this article is to analyze the temporal trends of cervical cancer mortality in Brazil and calculate the projection of mortality through to the year 2030. Deaths that occurred within the 1996-2010 period were analyzed (Mortality Information System). Mortality trend analysis utilized the Joinpoint regression, while Nordpred was utilized for the calculation of projections.For Brazil, decreasing trends were identified (APC $=1.7 \%$ CI95\%-2.2; $-1.1 p<0.05$ ). The Midwest region presented a significant reduction trend ( $A P C=-1.3 \%$ per year), along with the Southeast $(A P C=-3.3 \%)$ and South $(A P C=$ $-3.9 \%)$ regions. The North and Northeast regions presented stable trends. The states of Acre (APC $=-6.5 \%)$ and Rio Grande do Sul (APC $=-4.1 \%)$ presented the most pronounced reduction trends. Analysis of the mortality projections revealed a reduction in mortality rates, starting from the first projected period, with a considerable reduction for the South region. Mortality rates through to the year 2030 are explained, principally, by reductions in the riskof the disease. Cervical cancer mortality presents reducing trends, however these are unequally distributed throughout the country, where the North and Northeast regions present the highest mortality rates.

Keywords Cervical neoplasms, Mortality, Projections, Trends
Resumo $O$ objetivo deste artigo é analisar a tendência temporal da mortalidade por câncer de colo de útero no Brasil e calcular uma projeção até o ano de 2030. Foram analisados os óbitos ocorridos no Brasil de 1996 a 2010 (Sistema de Informações sobre Mortalidade). Foram realizadas análises das tendências da mortalidade por meio da regressão Joinpoint, e para o cálculo das projeções foi utilizado o Nordpred. Para o Brasil, a tendência é de redução (APC $=1,7 \%$ IC95\%$2,2 ;-1,1 p<0,05)$, sendo significativa nas regiões centro oeste ( $A P C=-1,3 \%$ ao ano), sudeste ( $A P C$ $=-3,3 \%)$ e sul $(A P C=-3,9 \%)$. As regiões norte e nordeste apresntam tendência de estabilidade. Os estados do Acre (APC = -6,5\%) e Rio Grande do Sul ( $A P C=-4,1 \%)$ apresentaram as maiores tendências de redução. Na análise das projeções de mortalidade, haverá uma redução das taxas no Brasil a partir do primeiro período projetado, sendo mais marcante para a região sul. As taxas de mortalidade até o ano 2030 serão explicadas, em maior medida, pela redução dos risco para a doença. A mortalidade por câncer de colo de útero apresenta tendência de redução, todavia está desigualmente distribuída no Brasil, com as regiões norte e nordeste apresentando as maiores taxas.

Palavras-chave Neoplasias do colo do útero, Mortalidade, Projeções, Tendências 


\section{Introdução}

A terceira causa de câncer mais incidente em mulheres em todo mundo é o de colo de útero, representando cerca de $9 \%$ dos casos, e nos países em desenvolvimento é a causa mais comum nesse grupo ${ }^{1}$. Com a estimativa de 529.000 casos e 275.000 óbitos por ano em todo o mundo, a carga do câncer de colo de útero varia consideravelmente entre os países, com mais de 85\% dos casos da carga global da doença distribuída nos países de baixa ou média renda ${ }^{2}$. A diferença entre as taxas de incidência observada entre os países e as diversas regiões do planeta reflete a prevalência da infecção pelo human papilloma vírus (HPV) e da qualidade e da cobertura dos programas de screening que utilizam o Papanicolaou como método de rastreamento ${ }^{3}$.

O entendimento de que, em geral, o câncer de colo de útero está associado à infecção por uma linhagem oncogênica do HPV gerou diversas correlações entre o comportamento e a incidência por essa doença ${ }^{4}$. A partir desse conhecimento, um fator importante foi a introdução de vacinas profiláticas para infecção pelo vírus HPV colocadas à disposição na última década ${ }^{3}$. Além disso, o uso de contraceptivos orais, as doenças sexualmente transmissíveis, a multiparidade, os múltiplos parceiros sexuais desprotegidos, a atividade sexual de início precoce e o tabagismo são considerados fatores de risco para o câncer de colo de útero ${ }^{5}$.

No Brasil, para o ano de 2014, foram estimados 15.590 novos casos de câncer de colo do útero com cerca de 5000 óbitos 6 . A ampliação de acesso, diagnóstico de qualidade e tratamento em tempo oportuno para o câncer de colo de útero estão incluídos entre os 16 Objetivos Estratégicos do Ministério da Saúde para o período 2011-20157.

Apesar das mudanças nos padrões de mortalidade por câncer resultarem de variações na incidência da doença e de seus principais determinantes, a mortalidade é também influenciada pelos casos fatais, que são determinados, por sua vez, pelo diagnóstico no início e disponibilidade de melhores tratamentos e cuidados. Então, torna-se claro que a mortalidade por câncer de colo de útero é um complexo indicador resultante de variações internacionais e, principalmente, da organização interna dos sistemas de saúde dos países.

Em vista da importância dessa doença e seu potencial para a ação dos sistemas de saúde, o monitoramento das tendências da mortalidade ao longo do tempo e o conhecimento da carga desta no futuro é de grande relevância para pla- nejar e avaliar as políticas de controle do câncer, assim como os métodos de triagem que possam ser implementados de forma mais efetiva para detecção precoce e tratamento, direcionadas às áreas de maior vulnerabilidade e risco9. Dessa forma, o objetivo deste estudo foi analisar as tendências temporais da mortalidade por câncer de colo de útero no Brasil, regiões e estados brasileiros no período de 1996 a 2010 e realizar as projeções de mortalidade até o ano de 2030.

\section{Métodos}

Estudo ecológico de série temporal, baseado em dados secundários coletados do Sistema de Informação sobre Mortalidade (SIM) do Departamento de Informática do Sistema Único de Saúde (Datasus). Foram analisados os óbitos decorrentes da neoplasia maligna de colo de útero no Brasil, regiões e estados brasileiros no período de 1996 a 2010.

Além da análise dos óbitos com a codificação C53 (Câncer de colo de útero, International Classification of Disases, $10^{\text {th }}$ Revision), foi realizada a distribuição daqueles registrados em porção não especificada (C55), considerando que parte destes teve como causa básica o câncer de colo de útero. O método aplicado neste estudo para redistribuir os casos de câncer de útero em porção não especificada foi distribuir proporcionalmente os óbitos não especificados para os cânceres de corpo de útero e de colo de útero, de acordo com ano, faixa etária e localização geográfica ${ }^{10,11}$.

Foi analisada a tendência temporal da mortalidade nesse período e calculadas as projeções por quinquênio para os períodos de 2011-2015, 2016-2020, 2021-2025 e 2026-2030.

Para analisar as tendências de mortalidade, foi realizada a análise de regressão Joinpoint, utilizando o software Joinpoint Regression Program (National Cancer Institute, Bethesda, Maryland, USA), Versão 4.1.0. O objetivo da análise foi identificar a ocorrência de possíveis joinpoints onde uma mudança significativa na tendência ocorra.

O método aplicado identificou joinpoints baseado no modelo com no máximo 3 pontos de mudança. O modelo final selecionado foi o mais ajustado, com o Annual Percentage Change (APC) baseado na tendência de cada segmento, estimando se esses valores são estatisticamente significativos a um nível de confiança de 95\%. Os testes de significância utilizados baseiam-se no método de permutação de Monte Carlo e no cálculo da variação percentual anual da razão, utilizando o logaritmo da razão. 
Para quantificar a medida resumo da tendência ao longo do período fixo pré-determinado, foi calculado o Average Annual Percentage Change (AAPC). O AAPC é calculado baseado na média ponderada dos coeficientes angulares da linha de regressão com pesos iguais ao comprimento de cada segmento ao longo do intervalo.

Na descrição, os termos "aumento" ou "redução" significam que a tendência é estatisticamente significativa $(\mathrm{p}<0,05)$. Para as não significativas, foi usado o termo "estável".

As predições foram feitas para cada período utilizando o modelo idade-período-coorte do programa Nordpred (Cancer Registry of Norway, Oslo, Norway), inscrito no programa estatístico R. Os dados foram compilados em blocos de 5 anos e o grupo de idade limite considerado para a análise foi o primeiro com mais de 10 casos para o período combinado.

Os resultados das predições estão apresentados por grupos de idade (20-29 anos, 30-49 anos, 50-69 anos e > 70 anos) e para o total de óbitos observados e esperados para cada período para o Brasil e as 5 regiões brasileiras. Para cada período, foram calculadas as taxas de mortalidade ajustadas com base na população padrão mundial para comparações globais, expressas por $100.000 \mathrm{mu}$ lheres por ano (ASW/100.000 hab $)^{12}$.

Foram calculadas as mudanças anuais no número de casos no último período projetado (2026-2030) comparado ao último observado (2006-2010), no qual a proporção desta ocorreu em termos dos riscos ou demográficos (tamanho ou estrutura da população). Esses dois componentes podem ser diferentes de zero e apresentar uma direção positiva ou negativa. O cálculo pode ser expresso como segue ${ }^{13}$ :

$$
\Delta_{\text {tot }}=\Delta_{\text {risk }}+\Delta_{p o p}=\left(N_{f f f}-N_{o f f}\right)+\left(N_{o f f}-N_{o o o}\right)
$$

onde $\Delta$ tot é a mudança total, $\Delta$ risk é a mudança em função do risco, $\Delta$ pop é a mudança em função da população, Nooo é o número de casos observados, Nfffé o número de casos projetados, e Noff é o número de casos esperados quando as taxas de mortalidade aumentam durante o período observado.

Os dados de população para o Brasil, os estados brasileiros e o distrito Federal utilizados no cálculo das taxas de mortalidade no período de 1996 a 2010 e para o das projeções até 2030, foram obtidas das informações dos Censos (2000 e 2010), Contagem (1996) e projeções intercensitárias segundo faixa etária e sexo, do Instituto Brasileiro de Geografia e Estatística (IBGE).

Para a produção dos mapas de distribuição das taxas de mortalidade observadas e projeta- das, divididas por quartis e por estado brasileiro, foi utilizado o programa Tabwin 3.6.

\section{Resultados}

De 1996 a 2010, foram registrados no Brasil 89.764 óbitos por neoplasia maligna de colo de útero. A taxa de mortalidade padronizada à população mundial para o Brasil variou de 8,04 óbitos/100.000 habitantes, no ano de 1996 a 6,36 óbitos/ 100.000 habitantes, no ano de 2010. As maiores taxas de mortalidade foram apresentada pelo estado do Amazonas (18,62 óbitos/100.000 hab, no ano de 2010), seguido dos estados de Roraima (16,39 óbitos/100.000 hab, no ano de 2010) e Amapá (17,55 óbitos/100.000 hab, no ano de 1996) (Figura 1).

$\mathrm{Na}$ série histórica das taxas de mortalidade para o Brasil, a análise pelo Joinpoint verificou tendência de redução (APC $=1,7 \%$ IC95\%$2,2 ;-1,1 \mathrm{p}<0,05)$. A tendência foi de redução significativa para a região centro oeste (APC $=-1,3 \%$ ao ano), sudeste ( $\mathrm{APC}=-3,3 \%)$ e sul (APC $=-3,9 \%)$, sem ocorrência de Joinpoints para referidas regiões. A região norte apresentou estabilidade das taxas; todavia, a região nordeste apresentou tendência de aumento até o ano de 2006, seguido de uma de estabilidade. Os estados do Maranhão $(\mathrm{APC}=7,1 \%)$ e Roraima (APC = $5,7 \%$ ) apresentaram as maiores tendências de aumento nas taxas entre todos os estados brasileiros, enquanto os estados do Acre $(\mathrm{APC}=-6,5 \%)$ e Rio Grande do Sul (APC $=-4,1 \%)$ as maiores de redução (Tabela 1).

Seis estados brasileiros apresentaram mudança na característica da tendência durante o período analisado: São Paulo, Paraíba, Amazonas, Sergipe, Pernambuco e Mato Grosso. Desses, apenas o estado de São Paulo apresentou tendência de redução quando analisado pelo AAPC (AAPC $=-3,7 \%$ IC95\% -5,3; -2,2 p < 0,05); os demais apresentaram tendência de estabilidade: Paraíba (AAPC $=3,8 \% \mathrm{p}=0,2)$, Amazonas $(\mathrm{AAPC}=1,4 \mathrm{p}=0,5)$, Sergipe $(\mathrm{AAPC}=2,7 \% \mathrm{p}=$ $0,1)$, Pernambuco (AAPC $=-2,4 \mathrm{p}=0,1$ ) e Mato Grosso (AAPC $=-3,1 \mathrm{p}=0,3)$. Observa-se que a região nordeste $(\mathrm{AAPC}=1,1 \%$; IC95\% $-0,1 ; 2,4$ $\mathrm{p}=0,1)$ apresentou tendência de estabilidade na variação anual das taxas de mortalidade.

A Tabela 2 apresenta as taxas ajustadas para o período observado e as projeções para os quinquênios de 2011-2015, 2016-2020, 2021-2025 e 2026-2030 para o Brasil e regiões. Nas análises dos dados para o Brasil, observa-se que haverá uma redução das taxas de mortalidade a partir 


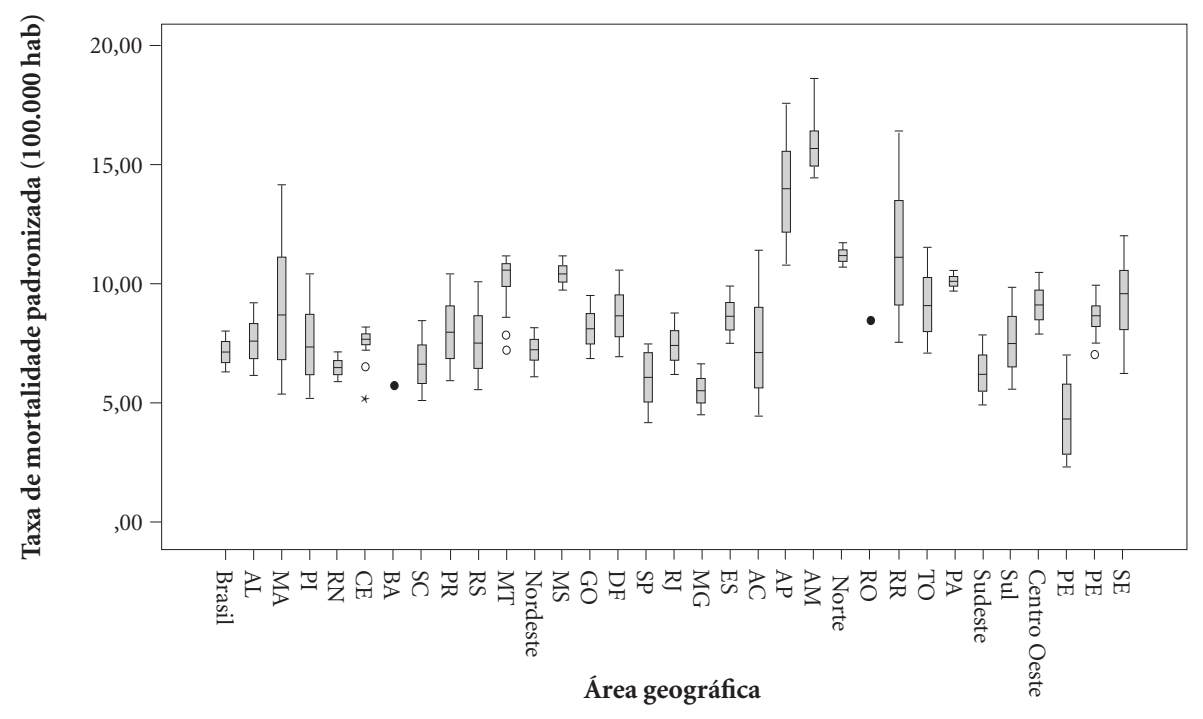

Figura 1. Taxas padronizadas de mortalidade por câncer de colo de útero para o Brasil e unidades federativas no período de 1996 a 2010. Brasil-2014.

do primeiro período projetado. Essa redução será mais marcante para a região sul, de $41,3 \%$ entre a última taxa observada e a última projetada, fazendo dessa região a menor no Brasil em 2030. Para o Brasil e todas as regiões brasileiras, o maior número de óbitos será registrado nas faixas etárias de 50 a 69 anos.

As variações nas taxas de mortalidade por estado brasileiro podem ser visualizadas na Figura 2, onde estão apresentados os quartis nos períodos observados e nos projetados. Nos períodos observados, as maiores taxas de mortalidade foram registradas nos estados do norte e parte do centro oeste e sul do Brasil. Ao longo dos períodos projetados, observa-se que haverá redução das taxas de mortalidade nos estados do sul, sudeste e centro oeste, com as maiores registradas nos das regiões norte e nordeste.

A Figura 3 mostra o percentual que os condicionantes 'fatores de risco' e 'mudanças demográficas' exercem na explicação para o número de óbitos no ano 2030. Esse cálculo mostrou que, em 2030, o número de óbitos esperados serão explicados em grande medida pela redução dos riscos para a doença, com exceção das regiões norte e nordeste, para as quais as mudanças demográficas explicarão o aumento das taxas de mortalidade até o ano 2030.

\section{Discussão}

Foi observada uma tendência de redução das taxas de mortalidade por câncer cervical para o Brasil no período compreendido entre 1996 e 2010, com importantes variações regionais. Comparadas às taxas de mortalidade por câncer de colo do útero das regiões de mais alta e baixa mortalidade do mundo, as do Brasil mostram-se em valores intermediários ${ }^{4,14,15}$.

$\mathrm{Na}$ análise do câncer cervical para 187 países, entre 1980 e 2010, foi observado que o número de casos tem aumentado na taxa de $0,6 \%$ ao ano, enquanto o número de óbitos tem crescido na proporção de $0,46 \%$ ao ano, gerado pelo aumento da população e as mudanças demográficas, respectivamente. Em diversas partes do mundo, a incidência e o risco da mortalidade por câncer de colo de útero tem decrescido.

Em pesquisa realizada para identificar a carga de câncer de colo de útero em 2008, as menores taxas de mortalidade foram observadas na Austrália (1,4 por 100.000 mulheres), na América do Norte (1,7 por 100.000 mulheres), e no Oeste Europeu ( 2 por 100.000 mulheres), enquanto que as maiores foram registradas no Leste da África (24 por 100.000 mulheres), Sudeste da África (14,8 por 100.000 mulheres) e na porção Sul-central da Ásia (10,8 por 100.000 mulheres $)^{1}$.

A tendência de redução da mortalidade por câncer cervical observada no presente estudo cor- 
Tabela 1. Tendência temporal da mortalidade por câncer de colo de útero no Brasil e regiões: Annual Percentage Change (APC), intervalo de confiança (IC 95\%) e ano do Joinpoint. Brasil, 2014.

\begin{tabular}{|c|c|c|c|c|c|c|c|c|}
\hline Unidade Federativa & APC1 & IC $95 \%$ & Joinpoint & APC2 & IC 95\% & Joinpoint & APC3 & IC 95\% \\
\hline Brasil & $-1,7^{*}$ & {$[-2,2 ;-1,1]$} & & & & & & \\
\hline Norte & 0,6 & {$[-0,2 ; 1,5]$} & & & & & & \\
\hline Nordeste & $2,9^{*}$ & {$[1,8 ; 4,0]$} & 2006 & $-3,2$ & {$[-7,2 ; 0,9]$} & & & \\
\hline Sudeste & $-3,3^{*}$ & {$[-3,9 ; 2,8]$} & & & & & & \\
\hline Sul & $-3,9^{*}$ & {$[-4,7 ;-3,0]$} & & & & & & \\
\hline Centro Oeste & $-2,0^{*}$ & {$[-3,6 ;-0,3]$} & & & & & & \\
\hline Acre & $-6,5^{*}$ & {$[-10,6 ;-2,2]$} & & & & & & \\
\hline Amapá & $-3,4^{*}$ & {$[-6,2 ;-0,5]$} & & & & & & \\
\hline Rondônia & 0,1 & {$[-3,0 ; 3,4]$} & & & & & & \\
\hline Roraima & $5,7^{*}$ & {$[1,6 ; 10,0]$} & & & & & & \\
\hline Pará & 0,6 & {$[-0,6 ; 1,8]$} & & & & & & \\
\hline Amazonas & $-0,9$ & {$[-7,7 ; 6,4]$} & 2003 & 3,7 & {$[-3,4 ; 11,3]$} & & & \\
\hline Tocantins & $3,5^{*}$ & {$[1,1 ; 6,1]$} & & & & & & \\
\hline Alagoas & $2,9^{*}$ & {$[0,3 ; 5,5]$} & & & & & & \\
\hline Sergipe & $7,5^{*}$ & {$[4,0 ; 11,1]$} & 2005 & $-5,4$ & {$[-12,7 ; 2,6]$} & & & \\
\hline Rio Grande do Norte & $-1,3$ & {$[-2,8 ; 0,3]$} & & & & & & \\
\hline Pernambuco & $-3,2^{*}$ & {$[-5,8 ;-0,5]$} & 2002 & 2,8 & {$[-5,2 ; 11,6]$} & 2006 & $-6,2^{*}$ & {$[-11,0 ;-1,2]$} \\
\hline Bahia & 0,4 & {$[-0,9 ; 1,7]$} & & & & & & \\
\hline Ceará & 0,8 & {$[-1,1 ; 2,7]$} & & & & & & \\
\hline Maranhão & $7,1^{*}$ & {$[4,8 ; 9,5]$} & & & & & & \\
\hline Paraíba & $-8,1$ & {$[-24,9 ; 12,5]$} & 1999 & $17,0^{*}$ & {$[9,3 ; 25,3]$} & 2006 & $-7,8$ & {$[-18,8 ; 4,8]$} \\
\hline Piauí & $5,1^{*}$ & {$[3,0 ; 7,2]$} & & & & & & \\
\hline São Paulo & 1,4 & {$[-6,4 ; 9,8]$} & 1999 & $-5,1^{*}$ & {$[-6,1 ;-4,1]$} & & & \\
\hline Rio de Janeiro & $-2,3^{*}$ & {$[-2,9 ;-1,7]$} & & & & & & \\
\hline Minas Gerais & $-2,7^{*}$ & {$[-3,4 ;-1,9]$} & & & & & & \\
\hline Espírito Santo & $-2,0^{*}$ & {$[-3,2 ;-0,7]$} & & & & & & \\
\hline Rio Grande do Sul & $-4,1^{*}$ & {$[-5,0 ;-3,2]$} & & & & & & \\
\hline Paraná & $-3,8^{*}$ & {$[-5,2 ;-2,5]$} & & & & & & \\
\hline Santa Catarina & $-3,4^{*}$ & {$[-4,5 ;-2,3]$} & & & & & & \\
\hline Mato Grosso & $-0,7$ & {$[-5,2 ; 3,9]$} & 2006 & $-8,6$ & {$[-24,2 ; 10,2]$} & & & \\
\hline Mato Grosso do Sul & $-0,9$ & {$[-2,6 ; 0,9]$} & & & & & & \\
\hline Distrito Federal & $-2,9^{*}$ & {$[-5,4 ;-0,4]$} & & & & & & \\
\hline Goiás & $-2,2^{*}$ & {$[-4,1 ;-0,3]$} & & & & & & \\
\hline
\end{tabular}

APC: annual percentage changes; 95\% CI, 95\% confidence intervals. *estatisticamente significativa p $<0.05$.

roborou os achados da pesquisa realizada para o estado de Minas Gerais (Brasil), que observou redução de cerca de $1,93 \%$ ao ano no período compreendido entre 1980-2005, com diminuição gradativa no período estudado, passando de 9,18/100 mil em 1980 para 5,70/100 mil em 2005, sendo também constatada uma redução da mortalidade para cada um dos estratos etários analisados ${ }^{16}$.

Tendências semelhantes foram encontradas em outros estudos que analisaram a mortalidade por esse câncer nos estados brasileiros. Em Rio Branco, no Acre, a mortalidade por essa neoplasia apresentou tendência decrescente: entre os anos de 1994 e 2000 observou-se diminuição acentuada na taxa, com variação anual percen- tual negativa de 10,7. Após esse período, a taxa se estabilizou em aproximadamente 11 mortes por 100 mil mulheres ${ }^{17}$. Todavia, quando analisados por estratos socioeconômicos, estudos realizados nos estados do Paraná ${ }^{18}$ e em São Paulo ${ }^{19}$ verificaram que para os estratos sociais que possuíam os piores indicadores socioeconômicos, a tendência na mortalidade era crescente.

Os países desenvolvidos reduziram em aproximadamente $80 \%$ o número de novos casos como resultado de um efetivo programa de detecção e tratamento das lesões pré-cancerígenas ${ }^{20}$. A exemplo dos Estados Unidos, que implementaram o teste Papanicolaou em meados da década de 1950, resultou na detecção precoce das lesões 
Tabela 2. Mortalidade por câncer de colo de útero no Brasil e regiões: número de óbitos observados e projetados por idade e taxa de mortalidade padronizada à população mundial (ASW/100.000 mulheres).

\begin{tabular}{|c|c|c|c|c|c|c|c|}
\hline & $1996-2000$ & 2001-2005 & 2006-2010 & 2011-2015 & 2016-2020 & 2021-2025 & 2016-2030 \\
\hline \multicolumn{8}{|l|}{ Brasil } \\
\hline 20-29 anos & 611 & 688 & 756 & 752 & 654 & 603 & 572 \\
\hline 30-49 anos & 9091 & 9533 & 9825 & 10151 & 10929 & 11900 & 12829 \\
\hline 50-69 anos & 11738 & 12691 & 13773 & 14498 & 15125 & 16084 & 17633 \\
\hline$\geq 70$ anos & 5918 & 6821 & 8319 & 9012 & 10198 & 11828 & 13789 \\
\hline Total & 27358 & 29733 & 32673 & 34413 & 36907 & 40415 & 44823 \\
\hline ASW & 7,74 & 7,26 & 6,48 & 5,85 & 5,43 & 5,21 & 5,17 \\
\hline \multicolumn{8}{|c|}{ Região Nordeste } \\
\hline 20-29 anos & 141 & 218 & 227 & 264 & 280 & 294 & 304 \\
\hline 30-49 anos & 2017 & 2488 & 2898 & 3524 & 4024 & 4392 & 4477 \\
\hline 50-69 anos & 2621 & 3345 & 4067 & 4869 & 5587 & 6258 & 6920 \\
\hline$\geq 70$ anos & 1320 & 1758 & 2767 & 3385 & 4243 & 5194 & 6091 \\
\hline Total & 6099 & 7809 & 9959 & 12042 & 14133 & 16137 & 17791 \\
\hline ASW & 11,07 & 10,91 & 11,72 & 12,37 & 12,74 & 12,84 & 12,69 \\
\hline \multicolumn{8}{|l|}{ Região Norte } \\
\hline 20-29 anos & 88 & 84 & 104 & 117 & 133 & 147 & 154 \\
\hline 30-49 anos & 787 & 973 & 1227 & 1551 & 1796 & 1980 & 2131 \\
\hline 50-69 anos & 757 & 937 & 1286 & 1707 & 2209 & 2750 & 3262 \\
\hline$\geq 70$ anos & 325 & 378 & 606 & 729 & 965 & 1293 & 1697 \\
\hline Total & 1957 & 2372 & 3223 & 4104 & 5103 & 6171 & 7244 \\
\hline ASW & 6,54 & 7,35 & 7,73 & 8,06 & 8,17 & 8,10 & 7,83 \\
\hline \multicolumn{8}{|c|}{ Região Centro Oeste } \\
\hline 20-29 anos & 39 & 61 & 76 & 64 & 57 & 54 & 51 \\
\hline 30-49 anos & 719 & 752 & 784 & 808 & 882 & 1004 & 1118 \\
\hline 50-69 anos & 852 & 949 & 1043 & 1083 & 1109 & 1165 & 1310 \\
\hline$\geq 70$ anos & 342 & 467 & 568 & 632 & 729 & 853 & 1014 \\
\hline Total & 1952 & 2229 & 2471 & 2587 & 2777 & 3075 & 3492 \\
\hline ASW & 10,08 & 9,55 & 7,91 & 6,64 & 5,82 & 5,40 & 5,28 \\
\hline \multicolumn{8}{|l|}{ Região Sudeste } \\
\hline 20-29 anos & 209 & 207 & 233 & 211 & 157 & 130 & 116 \\
\hline $30-49$ anos & 3675 & 3615 & 3391 & 3142 & 3243 & 3515 & 3900 \\
\hline 50-69 anos & 5307 & 5290 & 5260 & 5056 & 4916 & 5014 & 5449 \\
\hline$\geq 70$ anos & 2982 & 3142 & 3302 & 3208 & 3303 & 3616 & 4124 \\
\hline Total & 12173 & 12254 & 12186 & 11617 & 11619 & 12275 & 13589 \\
\hline ASW & 7,36 & 6,38 & 5,16 & 4,24 & 3,72 & 3,52 & 3,58 \\
\hline \multicolumn{8}{|l|}{ Região Sul } \\
\hline 20-29 anos & 134 & 120 & 118 & 100 & 74 & 60 & 51 \\
\hline 30-49 anos & 1901 & 1709 & 1524 & 1306 & 1267 & 1309 & 1404 \\
\hline 50-69 anos & 2199 & 2161 & 2078 & 1853 & 1672 & 1601 & 1660 \\
\hline$\geq 70$ anos & 932 & 1049 & 1039 & 1075 & 1134 & 1234 & 1380 \\
\hline Total & 5166 & 5039 & 4759 & 4335 & 4146 & 4203 & 4495 \\
\hline
\end{tabular}

pré-cancerígenas tratáveis e redução nas taxas de incidência da doença ${ }^{21}$.

No Brasil, as distintas características econômico-culturais, regionais e mesmo intraurbanas encontradas são capazes de gerar um padrão em que coexistem fatores relacionados à pobreza e ao desenvolvimento. A baixa condição socioeconômica interfere no acesso a serviços de prevenção, rastreamento regular, diagnóstico e tratamentos oportunos. Consequentemente, as limitações de acesso a serviços de saúde não somente impedem as mulheres pobres de serem diagnosticadas, mas também impossibilitam a oportunidade de receberem tratamento adequado a tempo de se obter a cura $^{22}$.

A distribuição de níveis hierárquicos de cuidado ao paciente com câncer dentro do Brasil é desigual, mostrando uma disparidade entre as áreas que têm melhores estruturas urbanas (regiões sudeste e sul), com a presença de siste- 

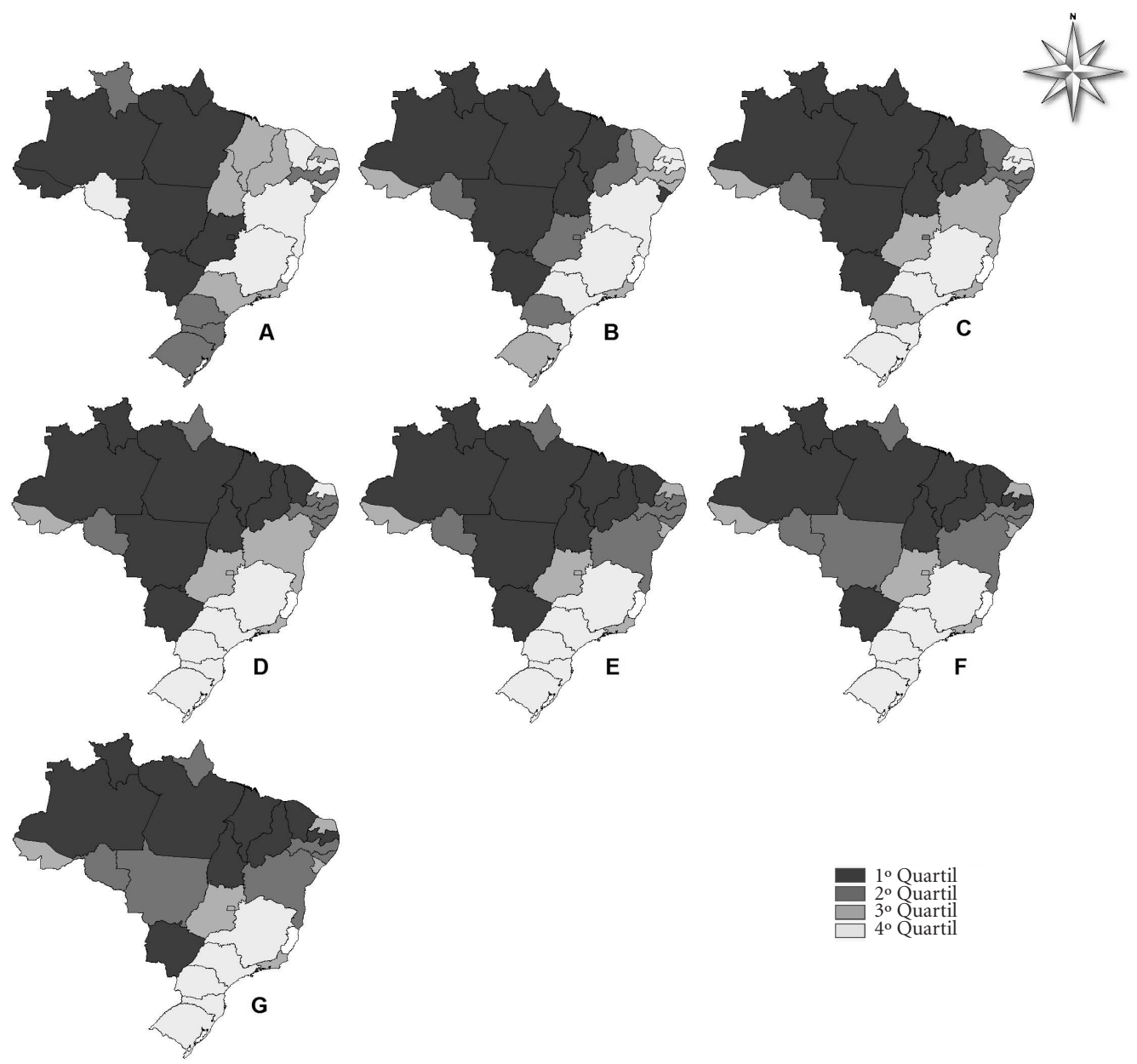

Figura 2. Quartis das taxas de mortalidade por câncer de colo de útero no Brasil nos períodos observados e projetados. Observados: A-1996-2000; B- 2001-2005. C- 2006-2010; Projetados: D-2011-2015; E- 2016-2020; F- 2021-2015; G- 2026-2030.

mas de saúde bem equipados e ordenadamente distribuídos no território, em contraponto com aquelas com ausência de níveis hierárquicos intermediários (no norte e nordeste). As regiões norte e centro oeste têm a ocupação do território de forma esparsa, o que impacta na organização e distribuição da oferta de serviços de saúde. Apesar da ocupação territorial consolidada no nordeste, há cidades que tradicionalmente concentram o aparato de equipamentos e serviços, com raros centros de cuidado em saúde de níveis intermediários ${ }^{23}$.

Estudos que avaliaram a tendência da mortalidade por câncer de colo de útero nas capitais brasileiras e nos municípios do interior dos estados mostraram que, notadamente, estes últimos apresentam padrão diferenciado de evolução e não indicam queda ou estabilidade nas taxas. Isso pode ser explicado, em parte, pelo menor alcance das ações de prevenção ao câncer nas cidades do interior e pela dificuldade de acesso a serviços de diagnóstico e tratamento para a população residente fora dos grandes centros urbanos. Sabe-se que a maior oferta de serviços de quimioterapia e radioterapia se concentra nas capitais das regiões sudeste e sul do Brasi ${ }^{24,25}$.

Para que haja um impacto epidemiológico na diminuição das taxas de incidência e mortalidade pelo CA de colo de útero, os programas de rastreamento devem cobrir 85\% das mulheres. No Brasil, a cobertura de tais programas foi descrita como sendo precária: cobre cerca de $8 \%$ a $10 \%$ da po- 


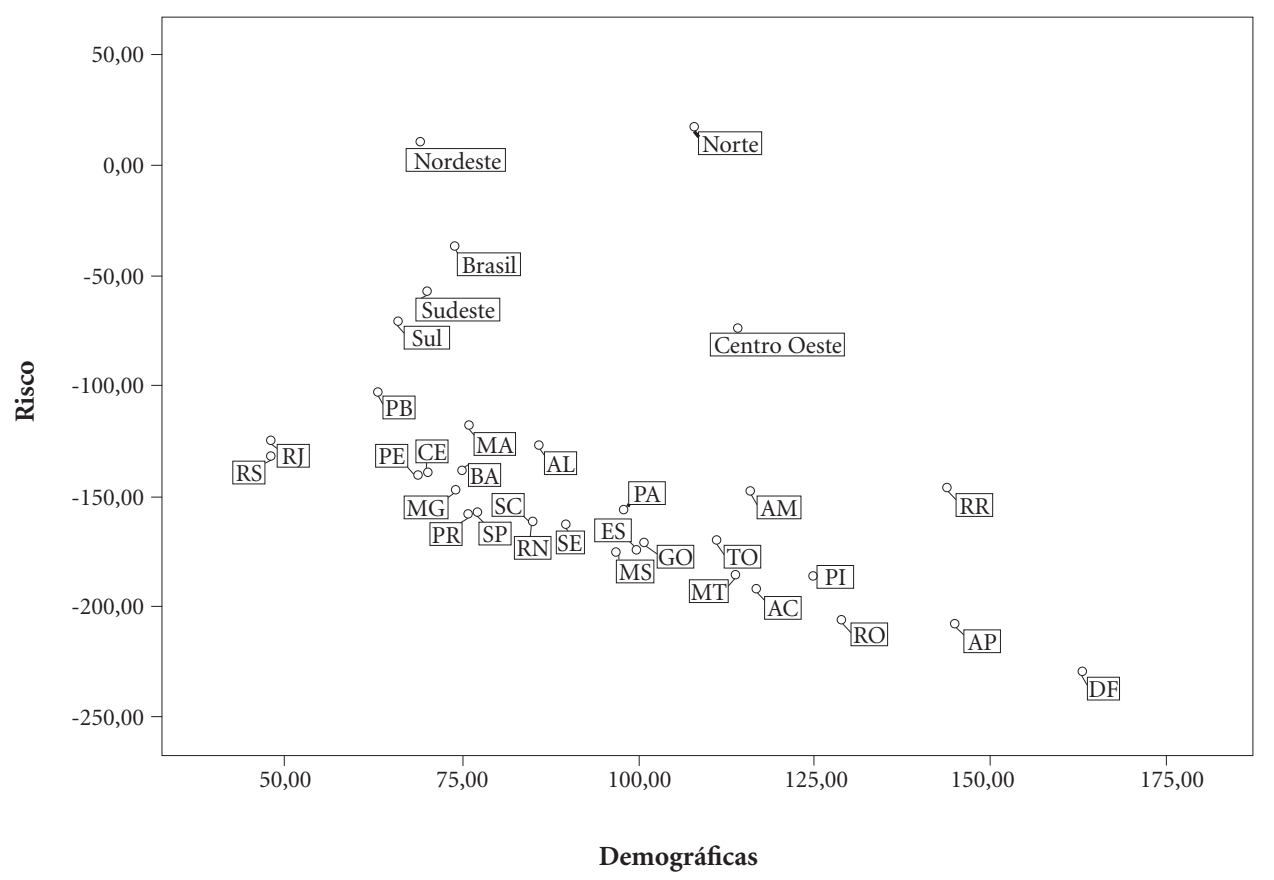

Figura 3. Mudança relativa devido ao risco e devido às mudanças na estrutura e tamanho da população na explicação das taxas de mortalidade em 2030. Brasil, 2014.

pulação feminina em torno dos 20 anos de idade ${ }^{18}$.

Em 1994, 37\% das mulheres brasileiras de 35 a 49 anos nunca haviam realizado o exame Papanicolaou. Após a implantação do Programa Nacional de Controle do Câncer do Colo do Útero em 1998, intensificou-se a oferta e foi ampliado o acesso ao exame de rastreamento. $\mathrm{O}$ resultado foi a ampliação da cobertura de 68,7\% em 2003 para $84,5 \%$ em 2008 do preventivo ginecológico entre mulheres com 25 anos ou mais ${ }^{26}$.

Na tentativa de estimar o número de exames Papanicolaou realizado por cada mulher na faixa etária de 25 a 49 anos, observa-se que em muitos estados brasileiros a média de 0,3 ao ano não é alcançada. Outro aspecto que deve ser analisado é a adequabilidade das lâminas, que em algumas análises por estado foram registrados percentuais acima de $5 \%$ insatisfatórias ${ }^{27}$.

A explicação para os achados deste estudo podem ser fundamentados na estruturação dos programas de triagem do câncer de colo de útero nas regiões brasileiras. Com o aumento da cobertura do exame citológico, dois fenômenos diferentes, intimamente ligados às diferenças nas condições de acesso, uso e desempenho dos serviços de saúde, podem ser observados. Por um lado, queda da mortalidade em regiões onde as condições sociais e de saúde podem garantir o tratamento e o seguimento para todas as pacientes com exames alterados, possibilitando a cura da doença. Por outro, aumento da mortalidade em regiões menos desenvolvidas, onde a cura não pode ser garantida, ou ainda onde a adesão da população-alvo é limitada, determinando que parte das pacientes sejam atendidas em fase já avançada da doença, com poucas possibilidades de $\mathrm{cura}^{28}$.

Além dos aspectos estruturais dos serviços de saúde, a prevalência dos fatores de risco na população pode ser determinante para explicar as taxas de mortalidade no presente e no futuro. No Brasil, estão registradas, pela Agência Nacional de Vigilância Sanitária, a vacina quadrivalente contra as linhagens do HPV 6, 11, 16 e 18, desenvolvida para a prevenção de infecção pelos tipos virais mais comuns nas verrugas genitais (HPV 6 e 11) e no câncer do colo do útero (HPV 16 e 18), e indicada para mulheres com idade de 9 a 26 anos; e a vacina bivalente contra HPV tipos 16 e 18, associados ao câncer do colo do útero, e indicada para as mulheres de 10 a 19 anos $^{27}$.

A incorporação da vacina contra HPV no Programa Nacional de Imunizações do Brasil deu-se a partir do ano de 2014 para o público alvo de meninas de 11 a 13 anos $^{29}$. Essa medida 
profilática poderá alterar as perspectivas futuras para a incidência e a mortalidade pelo câncer do colo do útero nos estados no Brasil, servindo de ferramenta ao controle desse tipo de câncer ${ }^{30}$.

De acordo com os resultados deste estudo, a projeção da mortalidade indica redução das taxas nas regiões sul, sudeste e centro oeste do Brasil. As regiões norte e nordeste apresentam tendência global de aumento das taxas ao longo dos 15 anos analisados, apesar desta última ter apresentado um ponto de mudança na tendência de mortalidade, com um descenso significativo nos últimos quatro anos, embora para confirmar a concretização dessa redução se necessite de uma série temporal mais longa para ser analisada. A perspectiva do aumento da mortalidade nas regiões mais pobres do Brasil revela a magnitude do desafio frente ao controle desse câncer no país.

O câncer de colo de útero, que está intimamente relacionado às regiões menos desenvolvidas e com os menores níveis socioeconômicos, requer a formulação de estratégias de controle como a organização e o desenvolvimento de serviços de saúde, mecanismos de formulação de políticas públicas e mobilização da sociedade ${ }^{31}$. Uma das consequências do aumento da mortalidade por esse câncer nas regiões mais pobres é o grande custo social e econômico que afeta os serviços de saúde já tão desestruturados.

A descentralização dos serviços de diagnóstico e os centros de referência para o tratamento do câncer reduziria a iniquidade no acesso e se configuraria numa importante estratégia de controle da incidência e da mortalidade. A tentativa de minimizar o impacto da mortalidade por esse câncer no Brasil, no futuro, deverá permear o adequado planejamento dos serviços, além da identificação dos centros de referência, a regionalização do tratamento, a redução das distâncias que os pacientes têm que se deslocar e a organização da demanda de tratamento por região ${ }^{32}$.

A mortalidade por câncer de colo de útero no Brasil apresenta tendência de redução significativa. A partir dos dados projetados até o ano de 2030, as taxas de mortalidade deverão continuar a apresentar esse padrão de redução. $\mathrm{O}$ quadro mais significativo são as diferenças regionais para a mortalidade por esse câncer, já que as regiões mais pobres do Brasil apresentam as taxas mais elevadas, enquanto as sul e sudeste, as mais desenvolvidas do Brasil, as mais baixas. Cabe ressaltar que essa discrepância será aprofundada até o ano de 2030, para o qual as regiões mais desenvolvidas deverão seguir reduzindo suas taxas, e, em contrapartida, as mais pobres, como o norte e nordeste do Brasil, deverão ter incremento. A partir da descrição desse cenário brasileiro, as políticas de saúde voltadas à compreensão desse fenômeno no Brasil poderão focalizar a observação de fatores de risco e traçar hipóteses para o controle da incidência da doença bem como do aparato necessário para reduzir as mortes consideradas evitáveis, tendo em vista que o carcinoma do colo do útero mostra incidência mais alta em populações urbanas que nas rurais, em classes sociais mais baixas do que nas mais altas, em áreas em desenvolvimento do que nas áreas mais desenvolvidas.

Uma das limitações deste estudo é o curto período de série temporal para realizar a análise de tendência e projeção de mortalidade: 15 anos. Sobre o registro de óbitos no Brasil, deve-se mencionar que no passado houve problemas com a confiabilidade dos dados, especialmente nas regiões norte e nordeste, porém, o ganho de qualidade e os avanços no sistema de informação sobre mortalidade vêm obtendo notoriedade desde o ano 2000. As projeções de câncer devem ser analisadas levando-se em consideração as atuais condições de diagnóstico e tratamento, as quais podem ser alteradas no futuro e, consequentemente, alterar as tendências de mortalidade. Entretanto, os métodos geralmente usados estão sendo validados e alguns autores pontuam que existam apenas diferenças entre $10 \%$ e $20 \%$ nas estimações.

\section{Colaboradores}

IR Barbosa planejou, coletou, analisou e interpretou os dados, e colaborou com a redação do artigo. DLB Souza planejou e interpretou os dados e colaborou com a redação do artigo. ICC Costa planejou e colaborou com a redação do artigo. MM Bernal interpretou os dados e colaborou com a redação do artigo.

\section{Agradecimentos}

À Coordenação de Aperfeiçoamento de Pessoal de Nível superior (CAPES), pelo financiamento deste projeto através do Programa de Doutorado Sanduíche no Exterior (PDSE), e ao Conselho Nacional de Desenvolvimento Científico e Tecnológico $(\mathrm{CNPq})$ pelo apoio. 


\section{Referências}

1. Arbyn M, Castellsagué X, de Sanjosé S, Bruni L, M. Saraiya M, Bray F, Ferlay J. Worldwide burden of cervical cancer in 2008. Annals of Oncology 2011; 22(12):2675-2686.

2. Vaccarella S, Lortet-Tieulent J, Plummer M, Franceschi S, Bray F. Worldwide trends in cervical cancer incidence: Impact of screening against changes in disease risk factors. Eur J Cancer 2013; 49(15):3262-3273.

3. D'Souza G, Cullen K, Bowie J, Thorpe R, Fakhry C. Differences in Oral Sexual Behaviors by Gender, Age, and Race Explain Observed Differences in Prevalence of Oral Human Papillomavirus Infection. PLoS ONE 2014; 9(1):e86023.

4. Dickinson JA, Stankiewicz A, Popadiuk C, Pogany L, Onysko J, Miller AB. Reduced cervical cancer incidence and mortality in Canada: national data from 1932 to 2006. BMC Public Health 2012; 12(992):1-13.

5. Kelava I, Tomičić K, Kokić M, Ćorušić A, Planinić P, Kirac I, Murgić J, Kuliš T, Znaor A. Breast and gynecological cancers in Croatia, 1988-2008. Croat Med J 2012; 53(2):100-108.

6. Instituto Nacional do Câncer (INCA). Estimativa 2014: Incidência de Câncer no Brasil. Rio de Janeiro: INCA; 2014.

7. Brasil. Ministério da Saúde (MS). Planejamento estratégico do Ministério da Saúde: 2011-2015: resultados e perspectivas. Brasília: MS; 2013.

8. Cabanes A, Vidal E, Pérez-Goméz B, Aragone N, Lópes-Abente G, Pollá M. Age-specific breast, uterine and ovarian cancer mortality trends in Spain: Changes from 1980 to 2006. Cancer Epidemiol 2009; 33(3-4):169-175.

9. Forouzanfar MH, Foreman KJ, Delossantos AM, Lozano R, Lopez AD, Murray CJL, Naghavi M. Breast and cervical cancer in 187 countries between 1980 and 2010: a systematic analysis. Lancet 2011; 378(9801):1461-1484.

10. Antunes JLF, Wünsch-Filho V. The effect of performing corrections on reported uterine cancer mortality data in the city of São Paulo. Braz Med Biol Res 2006; 39(8):1091-1099.

11. Loos AH, Bray F, Mccarron P, Weiderpass E, Hakama M, Parkim DM. Sheep and goats: separating cervix and corpus uteri from imprecisely coded uterine cancer deaths, for studies of geographical and temporal variations in mortality. Eur J Cancer 2004; 40(18):2794-803.

12. Doll R, Payne P, Waterhouse JAH. Cancer incidence in five continents. Geneva: Union Internationale Contre le Cancer; 1966. v. I.

13. Møller B, Fekjær H, Hakulinen T, Sigvaldason H, Storm HH, Talbäck M, Haldorsen T. Prediction of cancerincidence in thenordiccountries: Empirical comparison of different approaches. Stat Med 2003; 22(17):2751-2766.

14. Rodríguez-Rieiro C, Aragonés N, Pollan M, LópezAbente G, Pérez-Gomez B. Evolución de la mortalidad por cáncer de cérvix por regiones em España entre 1975 y 2004. Med Clin 2009; 133(2):41-46.

15. Kok IMCM, Van der A MA, van Ballegooijen M, Siesling S, Karim-Ko HE, Van Kemenade FJ, Coebergh JWW. Trends in cervical cancer in the Netherlands until 2007: has the bottom been reached? Int J Cancer 2011; 128(9):2174-2181.

16. Alves CMM, Guerra MR, Bastos RR. Tendência de mortalidade por câncer de colo de útero para o Estado de Minas Gerais, Brasil, 1980-2005. Cad Saude Publica 2009; 25(8):1693-1700.

17. Nakashima JP, Koifman S, Koifman RJ. Tendência da mortalidade por neoplasias malignas selecionadas em Rio Branco, Acre, Brasil, 1980-2006. Cad Saude Publica 2011; 27(6):1165-1174.
18. Müller EV, Biazevic MGH, Antunes JLF, Crosato EM. Tendência e diferenciais socioeconômicos da mortalidade por câncer de colo de útero no Estado do Paraná (Brasil), 1980-2000. Cien Saude Colet 2011; 16(5):2495-2500.

19. Fonseca LAM, Ramacciotti AS, Eluf Neto J. Tendência da mortalidade por câncer do útero no Município de São Paulo entre 1980 e 1999. Cad Saude Publica 2004; 20(1):136-142.

20. Alves CMM, Bastos RR, Guerra MR. Mortality due to cancer of the uterine cervix in the state of Minas Gerais, Brazil, 1980-2005:period and cohort analysis. Cad Saude Publica 2010; 26(7):1446-1456.

21. De Freitas AC, Gurgel APA, Chagas BS, Coimbra EC, Amaral CMM. Susceptibility to cervical cancer: An overview. Gynecol Oncol 2012; 126(2):304-311.

22. Rodrigues AD, Bustamante-Teixeira MT. Mortalidade por câncer de mama e câncer de colo do útero em município de porte médio da Região Sudeste do Brasil, 1980-2006. Cad Saude Publica 2011; 27(2):241-248.

23. Gonzaga CMR, Freita Júnior R, Barbaresco AA, Martins E, Bernardes BT, Resende APM. Cervical cancer mortality trends in Brazil: 1980-2009 Tendência da mortalidade por câncer do colo do útero no Brasil: 1980 a 2009. Cad Saude Publica 2013; 29(3):599-608.

24. Azevedo e Silva G, Girianelli VR, Gamarra CJ, Bustamante-Texeira MT. Cervical cancer mortality trends in Brazil, 1981-2006. Cad Saude Publica 2010; 26(12): 2399-2407.

25. Azevedo e Silva G, Gamarra CJ, Girianelli VR, Vlente JG. Tendência da mortalidade por câncer nas capitais e interior do Brasil entre 1980 e 2006. Rev Saude Publica 2011; 45(6):1009-1018.

26. Meira KC, Silva GA, Silva CMFP, Valente JG. Efeito idade-período-coorte na mortalidade por câncer do colo uterino. Rev Saude Publica 2013; 47(2):274-282.

27. Guimarães RM, Muzi CD, Ayres ARG, Ribeiro MS, Chagas CC, Oliveira JSC. Aplicação de Três Técnicas para Avaliação de Tendência de Mortalidade por Câncer do Colo do Útero em Série Temporal no Brasil, 1980-2009. Rev Bras Cancerol 2012; 58(3):359-367.

28. Gamarra CJ, Valente JG, Azevedo e Silva G. Magnitude da mortalidade por câncer do colo do útero na Região Nordeste do Brasil e fatores socioeconômicos. Rev Panam Salud Publica 2010; 28(2):100-106.

29. Brasil. Ministro da saúde lança campanha de vacinação contra o HPV [acessado 2014 jun 25]. Disponível em: www.brasil.gov.br.

30. Stark A, Gregoire L, Pilarski R, Zarbo A, Gaba A, Lancaster W. Human papillomavirus, cervical cancer and women's knowledge. Cancer Detect Prev 2008; 32(1):15-22.

31. Gonzalez-Robledo MC, Gonzalez-Robledo LM, Nigenda G. Formulación de políticas públicas sobre el cáncer de mama en América Latina. Rev Panam Salud Publica 2013; 33(3):183-189.

32. Knaul FM, Alleyne G, Piot P, Atun R, Gralow JR, Neal C, Sepulveda J, Frenk J. Health system strengthening and cancer: a diagonal response to the challenge of chronicity. In: Knaul FM, Gralow JR, Atun R, Bhadelia A, editors. Closing the cancer divide: an equity imperative. Cambridge: Harvard Global Equity Initiative; 2012. p. 79-95.

Artigo apresentado em 19/03/2015

Aprovado em 22/04/2015

Versão final apresentada em 24/04/2015 